Revue

de I'histoire des religions

\section{Revue de l'histoire des religions}

$4 \mid 2005$

Lieux de culte, lieux saints dans le judaïsme, le christianisme et l'islam

\title{
Fonctions et formes de la synagogue : refus et tentation de la sacralisation
}

Functions and Forms of the Synagogue. Temptations and refusal of sacralization

Dominique Jarrassé

\section{(2) OpenEdition}

\section{Journals}

Édition électronique

URL : http://journals.openedition.org/rhr/4216

DOI : $10.4000 /$ rhr.4216

ISSN : 2105-2573

Éditeur

Armand Colin

Édition imprimée

Date de publication : 1 octobre 2005

Pagination : 393-409

ISBN : 2200-92087-3

ISSN : 0035-1423

Référence électronique

Dominique Jarrassé, «Fonctions et formes de la synagogue : refus et tentation de la sacralisation », Revue de l'histoire des religions [En ligne], 4 | 2005, mis en ligne le 14 janvier 2010, consulté le 30 avril 2019. URL : http://journals.openedition.org/rhr/4216 ; DOI : 10.4000/rhr.4216 


\section{Fonctions et formes de la synagogue : refus et tentation de la sacralisation}

La synagogue est une fonction et non un type architectural défini par une tradition; aussi s'est-elle trouvée tiraillée entre deux modèles, le Temple de Salomon et l'église, qui ont tendu à lui imposer un caractère sacré, qu'elle n'avait pas originellement. Lieu d'assemblée d'une plasticité étonnante, la synagogue a emprunté les formes les plus variées aux cultures au sein desquelles elle s'est implantée. Avec l'émancipation, elle devient un "temple israélite» et le cadre d'une réforme qui se sert des formes inspirées des autres cultes pour imposer de nouveaux comportements aux fidèles, séparant surtout les espaces de culte et d'assistance, et magnifier sa fonction cultuelle. Il faut attendre l'adoption des esthétiques modernistes pour que la synagogue, intégrée dans un centre communautaire, retrouve sa simplicité initiale.

\section{Functions and Forms of the Synagogue. Temptations and refusal of sacralization}

The synagogue's nature is functional. It is not an architectural design determined by tradition. It has therefore been torn between two models: Solomon's temple and the church, both leading on to a character sacred that did not exist originally. Places of gathering, surprisingly plastic, synagogues borrowed a multitude of forms from the cultures within which they had been implanted. When emancipation took place, the synagogue became an "Israelite temple" and the frame for a reform using forms inspired by other worships to impose new behaviours to the faithfuls, mostly setting apart spaces devoted to worship and those devoted to welfare, and magnifying its cultual function. It is only with the adoption of modernist aesthetics that the synagogue, integrated in a community center does recover its initial simplicity. 
En quête d'une catégorie qui puisse englober les édifices élevés par diverses confessions, l'historien qui recourt à celle de sacré, risque de méconnaître le sens de la synagogue qui n'est ni un lieu saint, ni un sanctuaire, mais tout au plus un lieu de culte, et encore à certaines nuances près. Cependant, par des processus d'assimilation compréhensibles, la référence à ces désignations peut se rencontrer et nous verrons que même chez les Juifs, la tentation existe de valoriser la fonction cultuelle de la synagogue, par souci de dignité ou par revendication d'égalité face aux autres confessions. Si la désignation hébraïque de la synagogue, bet haknesset, qui signifie, comme le grec sunagôgê, maison d'assemblée, tend évidemment à l'identifier avec l'ecclesia, sa nature et sa fonction s'en différencient profondément. De toute manière, l'espace synagogal se définit en premier lieu par la présence de dix hommes de plus de treize ans, le minian $^{1}$, quorum nécessaire pour que le culte public puisse se dérouler; le rassemblement des hommes importe plus que le lieu physique qui n'est donc pas l'objet d'une quelconque consécration. Inaugurer ${ }^{2}$ une synagogue consiste seulement à installer dans l'arche sainte un rouleau de la Tora. Évidemment, à cette occasion, les rabbins tenus de faire une allocution ne manquent pas d'évoquer la prière de consécration du Temple prononcée par Salomon (I Rois, VIII, 22-53), mais ce rapprochement n'induit pas une identité de statut de ces espaces.

Il est par ailleurs impossible d'envisager la synagogue dans la variété des formes qu'elle a prises au cours des âges et au contact des civilisations diverses qui l'ont imprégnée ; on ne peut qu'en esquisser quelques aspects majeurs, car évidemment une synagogue n'adopte pas impunément des formes puisées dans d'autres lieux de cultes. Irréductible à un type et marquée par une plasticité étonnante, la synagogue doit donc être considérée en premier lieu comme un espace fonctionnel ; dans certaines circonstances, néanmoins, elle a pu être sacralisée, le plus souvent par un recours à l'esthétique. Ce

1. Le mot signifie «nombre » et a fini aussi par désigner les oratoires.

2. Terme récusé évidemment par ceux qui retiennent son sens étymologique, puisqu'il laisse entendre une intervention des « augures » pour consacrer un lieu, et son sens ancien de « sacrer». 
processus justifie le point de vue de l'historien de l'art, mais ne saurait prétendre cerner totalement la question de la synagogue.

\section{UN ESPACE FONCTIONNEL ET SYMBOLIQUE, MAIS PAS SACRÉ}

La synagogue est une création historique, une invention humaine grandiose. Contrairement à la Tente d'assignation, Ohel moed, elle n'est pas instituée par Dieu dans le texte révélé ; de même, elle n'a pas la sacralité du Temple, bet hamiqdach, construit à Jérusalem par David et Salomon et investi par la Présence divine. Même si, après son apparition présumée en Babylonie en lien avec la destruction du premier Temple, elle est qualifiée de «petit sanctuaire », miqdach meat, par Ezéchiel (XI, 16), elle ne peut en être considérée comme un véritable substitut, la mutation d'un édifice à l'autre étant trop profonde. Selon les paroles d'Osée XIV, 3 : «nous voulons remplacer les taureaux par cette promesse de nos lèvres », à un sanctuaire fondé sur des sacrifices et organisé en différentes cours, le Temple lui-même n'étant accessible qu'à une caste sacerdotale, a succédé une salle de réunion où se déroulent des lectures, des commentaires, des prières, mais aussi des activités communautaires ; aux prêtres a succédé un "peuple de prêtres », c'est-à-dire l'ensemble des Juifs assumant la transmission à travers l'étude. Le Talmud a voulu établir une continuité symbolique entre le Temple et la synagogue : ainsi il conserve le souvenir du roi Joachin (Yehoyakhin) emportant à Babylone des pierres du Temple pour s'en servir dans la construction d'une synagogue ${ }^{3}$. Le statut de la synagogue est profondément lié à ce contexte exilique, puis de diaspora. Comme le proclamait en chaire un grand rabbin français ${ }^{4}:$ "C'est la synagogue, mes frères, qui a, en quelque sorte, élaboré, fondé, créé le judaïsme ! Et il citait un midrach sur le Cantique des cantiques : «J'ai tout perdu, car mon sanctuaire est détruit... Je n'ai rien perdu, car il me reste

3. Meguila, 29a.

4. Zadok Kahn, «Le Temple. Schemini-Atsereth 5633 », prononcé le 24 octobre 1872, Sermons et allocutions, $1^{\text {re }}$ série, $2^{\mathrm{e}}$ édition, Paris, Durlacher, 1893 , p. 159. 
des maisons de prière et des maisons d'études. ${ }^{5}$ Le grand rabbin met l'accent, à travers ces mots, sur les fonctions principales de la synagogue: bet hatefila, maison de prière, et bet hamidrach, maison d'étude.

La disparition du Temple, centre spirituel, cultuel et politique du judaïsme jusqu'en 70 de notre ère, et la dispersion obligent les Juifs à inventer une nouvelle forme de religion dont les actes essentiels se déroulent à la maison, elle-même petit sanctuaire, et dans la salle d'assemblée. Dans ce dernier lieu, la communauté se rassemble pour écouter la Loi et les commentaires assurés par des rabbins : la fonction d'étude, bet hamidrach, est tellement primordiale que dans certains pays, la synagogue est désignée comme scuola ou schule, école. Moins vitale pour la survie de la communauté qu'un mikvè, bain rituel, moins valorisée que la maison d'étude, la synagogue ne donne pas lieu à des prescriptions talmudiques très précises $^{6}$. Quelques principes pratiques, qui semblent plutôt la consécration d'usages traditionnels, sont édictés, mais la plupart, en dehors de la mehitsa ${ }^{7}$, ont surtout une valeur symbolique. Ainsi l'orientation de l'arche sainte vers Jérusalem est recommandée, mais en cas d'impossibilité l'édifice n'en est pas moins utilisable, les fidèles se tournant pour certaines prières. Nombre de prescriptions symboliques, comme celle qui voudrait que la synagogue soit l'édifice le plus élevé de la ville, ne purent presque jamais être suivies. Il faudra la réaction orthodoxe du XIX ${ }^{\mathrm{e}}$ siècle, face aux tendances réformistes, pour que le respect de certaines règles devienne impératif.

Malgré cette souplesse dans sa construction, une synagogue reçoit un certain degré de sainteté en raison de la présence des rouleaux de la Tora dans l'armoire justement qualifiée d'aron hakodech,

\section{Ibid., p. 147.}

6. Le Choulhan Aroukh, code rédigé par Joseph Caro au XVI ${ }^{\mathrm{e}}$ siècle, rappelle qu'une synagogue peut être convertie en maison d'étude et non l'inverse. Nous citons d'après la version d'Ernest Weill, Choulhane Aroukh abrégé, Fondation Sefer, Paris, 1980, p. 81-82.

7. "Séparation», ici des hommes et des femmes, qui a nécessité la construction de pièces particulières pour les femmes; dans la plupart des synagogues modernes, elle est obtenue par une surélévation ou une claire-voie. 
arche sainte, ou dans certaines traditions, hekhal, mot désignant la salle du Temple où se dressaient le chandelier, la table des pains et l'autel des encens. Deux traits traduisent ce caractère de sainteté. D'une part, l'attitude de respect des fidèles qui doivent couvrir leur tête, et dans quelques pays orientaux, se déchausser, comme les prêtres dans le Temple; des inscriptions talmudiques appropriées, des panneaux ornés ${ }^{8}$ et une décoration plus abondante rappellent la sainteté de l'armoire d'autre part. On trouve également des motifs symboliques puisés dans le Temple, comme le rideau (parohet) qui séparait le Saint du Saint des Saints et qui est repris devant l'aron hakodech.

La primauté de la fonction pratique se lit clairement dans le fait que seulement deux éléments de mobilier sont essentiels dans la synagogue : l'arche sainte et une estrade de lecture (bima). Leur disposition induit les plans possibles et leur relation est significative. L'arche doit être plus élevée que l'estrade, afin de suivre la suggestion du psaume 130. Longtemps, cette armoire fut amovible et elle ne se fixa sur le mur tourné vers Jérusalem que progressivement : une niche, sans doute issue du modèle de la basilique, a servi à la mettre en valeur, puis un édicule toujours plus monumental. Quant à l'estrade, elle a pris des formes variées, surtout en hauteur, car on « monte » à la Tora, mais elle obéit traditionnellement à une règle qui associe le côté pratique et la valeur symbolique, la centralité. En étant située au milieu de la synagogue, la bima permet la circulation aisée des fidèles qui viennent tour à tour officier et une meilleure audition. Certaines processions des rouleaux de la Tora peuvent également se dérouler facilement autour, attestant déjà la signification profonde de cette disposition concentrique, rappel du mont Sinaï : quand Moïse reçut les tables de la Loi, le peuple était assemblé autour de la montagne. Enfin, il est précisé dans Néhémie (VIII, 3-5) qu'Ezra lut la Tora devant le peuple assemblé « sur une estrade en bois qu'on avait élevée pour la circonstance ». Un rituel synagogal semble y trouver une origine, hagbaha, une élévation du rouleau avant (rite séfarade) ou après (achkenaze) la lecture :

8. Le cheviti (premier mot du verset 8 du psaume XVI : « Je fixe constamment mes regards sur le Seigneur ») prend souvent la forme d'un tableau. 
«Ezra ouvrit le livre aux yeux de tout le peuple, car il dominait tout le peuple». Ces éléments témoignent d'un temps, celui de la reconstruction du second Temple, où officiaient encore des prêtres, mais où la lecture de la Tora et son commentaire étaient devenus aussi une part de la vie religieuse. Il a fallu toute la puissance du modèle chrétien séparant assistance et officiants, pour qu'au $\mathrm{XIX}^{\mathrm{e}}$ siècle, la bima soit rapprochée de l'arche sainte dans une sorte de chœur, une réforme refusée par les Juifs séfarades et orientaux, comme par les Achkenazes orthodoxes. Pourtant seuls des responsa rabbiniques prescrivent cette mesure fonctionnelle et hautement symbolique.

La force du modèle chrétien est telle qu'on en arrive à oublier que l'esthétique n'est pas obligatoirement une traduction du sacré, mais une manière d'honorer l'Éternel. Une autre idée reçue, parallèle à l'interprétation tronquée du $2^{\mathrm{e}}$ commandement qui prohiberait l'image, est celle qui voit dans les Juifs un peuple porteur d'une éthique au détriment d'une esthétique. C'est un fait indéniable, car toute religion subordonne l'esthétique à l'éthique, mais la primauté accordée aux valeurs morales n'exclut pas l'usage du Beau. Les Juifs peuvent avoir privilégié le temps, selon la métaphore célèbre d'Abraham Heschel qui les voit en Bâtisseurs du temps (1957), mais n'ont pas pour autant pu se dispenser de construire dans l'espace, une société, des structures urbaines et même des monuments. Toutefois, si la monumentalité n'est pas nécessaire à la synagogue, l'esthétique n'en est pas absente, en raison de l'impératif de hiddour mizwa, c'est-à-dire le devoir d'honorer Dieu en magnifiant tout acte et la réalisation de tout devoir par une dimension esthétique. Le Talmud prescrit: «Ayez le souci du beau dans l'accomplissement de vos cérémonies religieuses $»^{9}$. De même, il propose l'interprétation du passage de l'hymne de Moïse après le passage de la Mer rouge "voilà mon Dieu, je lui rends hommage » (Exode, $\mathrm{XV}, 2)$ en « je veux le rendre beau ${ }^{10}$, c'est-à-dire, poursuit le Choulhane Aroukh, « en me présentant devant Lui avec des objets du culte, je choisis les plus

9. Houlin, 44b.

10. Chabbat, 133b. 
beaux ${ }^{11}$. Il convient que l'objet d'une mizva soit « de toute beauté », quitte à ce qu'il coûte un tiers plus cher ${ }^{12}$.

La valeur esthétique n'est donc pas ignorée, mais elle ne saurait être qu'une modalité du bon : ainsi Balaam, devant les tentes d'Israël, prononce cette bénédiction : Ma tovou... (Nombres, XXIV, 5) qu'il est traditionnel de traduire par : «Qu'elles sont belles, tes tentes, ô Israël... », mais, en vérité, le mot tov signifie «bon ». Cependant, la monumentalité et l'esthétique s'imposèrent, lorsque la synagogue devint, en diaspora, non seulement le lieu de réunion, mais l'image même de la communauté. Cette fonction de représentation sociale a favorisé des emprunts aux autres édifices de culte, qui ont pu se lire comme une sacralisation.

\section{SACRALISATION DE LA SYNAGOGUE AU XIX ${ }^{\mathrm{e}}$ SIÈCLE}

Au cours du temps, si la fonction de la synagogue demeure à peu près inchangée, sa forme a connu des évolutions notables, strictement sur le plan de l'enveloppe. Les réalisations ou la quête d'un style, dans le contexte du XIX ${ }^{\mathrm{e}}$ siècle, ont amené les architectes à proposer des options inspirées d'autres édifices de culte et à surajouter fréquemment une dimension de sacralité par le recours à des formes symboliques.

Comment ne pas imaginer que la synagogue est un lieu saint quand elle prend la forme d'une cathédrale ? Un ingénieur berlinois, Ernst Hiller, notait en 1906 dans la revue Ost und West : « On est surpris, quand entrant dans une synagogue, on a la même impression qu'en entrant dans une église chrétienne. La construction, l'organisation de l'espace et les formes paraissent absolument chrétiennes. Qu'à l'emplacement où dans l'église se trouve l'autel, la disposition soit quelque peu différente, ne saute aux yeux qu'en le considérant de plus près. Chœur, orgue, chaire, la situation est tout à fait chrétienne. ${ }^{13}$

11. Op. cit., chapitre CCL, 8, p. 625.

12. Ibid., XXXIV, 5, p. 193.

13. Ernst Hiller, "Betrachtungen über den modernen Synagogenbau », Ost und West, $6^{\mathrm{e}}$ année, $\mathrm{n}^{\circ} 1$, janvier 1906. 
Cette christianisation des formes de la synagogue, devenue « temple israélite ${ }^{14}$, n'est pas sans impact sur les comportements, donc sur le rituel. Le premier facteur de cette transformation est la promotion de la synagogue en lieu de culte : on essaie d'éliminer les autres fonctions, reléguées dans des annexes, pour magnifier l'espace où se déroule un rituel chargé de décorum. Des orgues, des chaires, des costumes, des "enfants de chœur», une ordonnance des cérémonies, parallèlement à l'élimination de pratiques jugées désormais déplacées comme la vente aux enchères des places ou des mizwot (les honneurs attachés à la lecture de certains textes à des moments importants du rite ou de l'année liturgique), viennent entériner l'idée que règne dans la synagogue un ordre entièrement bâti en vue de donner une image positive, d'atteindre à la dignité des autres confessions ; ainsi l'atmosphère de plus d'une synagogue a fini par ressembler à celle des temples protestants.

Le second facteur, de nature architecturale, en découle : le rituel doit s'adapter à l'espace élaboré en fonction des modèles, et non l'inverse. Il est flagrant que l'architecture devient alors un moyen d'imposer de nouveaux comportements, un instrument de réforme. Ainsi, malgré son caractère peu adéquat pour conserver la bima au centre, c'est le plan basilical qui se répand le plus. On ne doit pas s'étonner qu'au bout d'un moment les architectes aient suggéré, pour des raisons de commodité et de gain en sièges, de déplacer la bima dans un chœur dévolu aux officiants. Ce réaménagement intérieur est un point crucial de la réforme qui agita les communautés d'Europe occidentale et d'Amérique du Nord au XIX siècle : il concrétise la scission entre espace dévolu à l'assemblée, à l'assistance, et espace « sacralisé » où officient des spécialistes ; ce dernier espace est mis en valeur par une séparation symbolique, une balustrade ou une surélévation... Le vocabulaire employé par les architectes alsaciens des années 1860 est significatif : l'un applique, selon les

14. D. Jarrassé, « Le temple israélite. Approche comparative d'un symbole et d'un instrument de l'israélitisme dans l'Europe et l'Amérique du XIX ${ }^{\mathrm{e}}$ siècle ", colloque Israélites et israélitisme. Espaces sociaux et courants d'idées, du XIXe siècle à nos jours, FRE CNRS Diasporas, Toulouse, 12-14 novembre 2003. Paru sous le titre Un modèle d'intégration, Berg International, 2004, p. 65-75. 
recommandations du Consistoire israélite de Strasbourg, une disposition intérieure qui consiste à « réunir au tabernacle la tribune du ministre-officiant, de manière à former au fond de la nef un sanctuaire où se trouvent réunis les différents services relatifs au culte ${ }^{15}$; il ajoute : "Cette disposition permet à tous les assistants de voir le tabernacle. » Un autre architecte explicite les principes imposés en ces termes ${ }^{16}:$ : 1 ) Reporter l'Almemer ${ }^{17}$ ou estrade des chantres vers le fond de la synagogue devant le tabernacle afin de donner à l'intérieur du temple plus de dignité ; 2) disposer les bancs de manière à tourner tous les assistants vers le tabernacle ; 3) à placer le tabernacle plus en évidence, en établissant du côté Est dans la largeur du bâtiment un perron... » L'emploi du mot «assistants » qui ne choque pas en milieu chrétien, montre que la tradition juive est en passe de se transformer en participation prioritairement visuelle; les architectes se soucient que les fidèles «voient » bien le chœur, non plus qu'ils « montent » à la Tora. De même, le rabbin doit désormais monter en chaire pour accroître sa dignité ; ainsi en avaient décidé les membres du Consistoire israélite de Strasbourg qui refusaient de transmettre au Ministère des Cultes les projets de synagogues, relevant de leur ressort, qui n'en avaient pas prévu ${ }^{18}$.

Toutes ces mesures se retrouveraient dans les synagogues depuis la Hongrie jusqu'à San Francisco, en passant par l'Angleterre ${ }^{19}$. Elles attestent une promotion de la synagogue qui se situe à deux

15. Timothée-Guillaume Roehrich, Synagogue de Hatten, rapport du 11 août 1865, Archives départementales du Bas-Rhin, V 529. Nous soulignons le mot « sanctuaire» dont l'emploi trahit la valorisation de cet espace.

16. Antoine Ringeisen, Mackenheim. Projet de construction d'une synagogue, 20 septembre 1864, Archives municipales de Sélestat, Fonds Ringeisen.

17. Ce mot, almemer ou almemor, désigne en Alsace la bima; il est intéressant de remarquer qu'il provient de l'arabe al-minbar, la chaire à prêcher de la mosquée.

18. Ses instructions du 3 juillet 1843 comportent un article 13 qui impose la chaire ; il n'était même pas tenu compte de l'étroitesse de certaines synagogues de campagne où la chaire ne s'imposait pas.

19. Sharman Kadish consacre justement un article aux "Cathedral Synagogues of England", Jewish Historical Studies, vol. 39, 2004, p. 45-77. Elle signale aussi l'emploi de choral synagogue pour décrire ces édifices avec chœur. 
niveaux, d'une part, comme nous venons de l'envisager, l'aménagement intérieur qui impose de nouveaux comportements, et d'une part, la valorisation de la fonction synagogale par référence aux autres lieux de culte et à l'édifice prestigieux qui passe pour leur ancêtre commun, le Temple de Jérusalem. Un double processus de transfert semble se produire entre le Temple de Jérusalem et la synagogue, censée en descendre : à la sécularisation du Temple ${ }^{20}$, dont la perception (et la reconstitution) se fait désormais à travers l'archéologie, et non plus la mémoire ritualisée, correspond une sacralisation de la synagogue ${ }^{21}$ qui va emprunter au premier des symboles sacralisants : comment expliquer, sinon, l'insistance avec laquelle les architectes emploient des motifs architecturaux du Temple ? Le meilleur exemple en est la transposition des colonnes Yakhin et Boaz : Ludwig Förster s'y réfère explicitement pour justifier les deux tours (en forme de minarets) qu'il place en façade de la synagogue de Budapest (1853-1858) et les deux piliers de l'entrée de la synagogue de Verdun (Henri Mazilier, 1875) offrent sur leurs chapiteaux les noms de ces colonnes en hébreu... Combien d'arches saintes ont reçu des symboles repris du Temple ou puisés dans le costume du grand prêtre Aaron, pectoral ou bandeau ! La reproduction de ce dernier, avec l'inscription kodech l'Adonä̈, montre bien par l'usage du mot kodech la valorisation de ce meuble empruntée à l'époque biblique.

Or, si la synagogue, dans son fonctionnement, est foncièrement différente du Temple, l'église en descend bien par plus d'un aspect. Cependant, ce n'est pas sans une contradiction, presque choquante, que la symbolique chrétienne se retrouve parfois employée pour sacraliser la fonction synagogale. Ainsi, il n'est pas rare de rencontrer

20. Cette transformation en «monument historique » nous paraît engagée dès le $\mathrm{XVIII}^{\mathrm{e}}$ siècle, lorsque les architectes s'efforcent d'intégrer le Temple dans l'histoire de l'architecture, au même titre que les merveilles du monde antique et les édifices grecs et romains sur lesquels furent établis les ordres. Ainsi le recueil de Johann Bernhard Fischer von Erlach, Entwurf einer historischen Architektur (1721), s'ouvre sur les planches consacrées au Temple.

21. D. Jarrassé, «Les mémoires du Temple. Sécularisation du Temple et sacralisation de la synagogue dans la culture juive française du XIX ${ }^{\mathrm{e}}$ siècle », Transmission et passages en monde juif, Paris, Publisud, 1997, p. 473-485. 
au XIX ${ }^{\mathrm{e}}$ siècle des synagogues avec un transept, or nul n'ignore que c'est là la représentation de la croix sur laquelle le Christ a été sacrifié. Néanmoins, par exemple, un architecte juif allemand, comme Edwin Oppler, qui, dans un souci d'intégration, prônait l'adoption des formes chrétiennes, adopte ce type de plan en croix pour les synagogues de Hanovre (1864-1870) ou de Wroclaw (Breslau, 1865-1872). En France, le cas le plus étonnant se rencontre à Dijon (Alfred Sirodot, 1879) où la croisée du transept est aussi surmontée d'une coupole, dans un vocabulaire romano-byzantin. On peut toujours interpréter la coupole comme symbole de l'unité divine, elle n'en coiffe pas moins une croix...

Une telle situation découle, en fait, non pas évidemment d'un choix délibéré de christianiser l'édifice, mais de l'absence de conscience que les styles historiques, utilisés pour contribuer à la nationalisation des communautés, ont été élaborés le plus souvent au service de l'Église. La synagogue, dans son incroyable capacité à s'adapter à toutes les formes, finit par en accepter qui véhiculent évidemment un sens qui ne peut lui convenir. Une des réponses à cette difficulté fut le recours à l'orientalisme : une référence à l'architecture mauresque semblait permettre d'échapper au modèle chrétien et signifier en même temps une origine orientale spécifique. Ce n'est pas un hasard, ce sont les synagogues orientalistes qui comportent le plus de références explicites au Temple de Jérusalem (certaines transitant peut-être même par le Dôme du Rocher...) Pour autant, il n'était guère plus sensé de confondre la synagogue avec la mosquée, comme à Berlin rue Oranienburg (Eduard Knoblauch, 1856-1866) ou à Besançon, sur les bords du Doubs (Pierre Marnotte, 1869).

\section{LA VARIÉTÉ DES FORMES}

Le principe qui préside à la conception des synagogues, sur le plan architectural, est, comme nous l'avons dit, un processus d'intégration, d'où leur extrême diversité. Longtemps les formes de la synagogue ont simplement découlé du contexte culturel dans lequel se situaient les communautés: les synagogues gréco-romaines 
présentaient des ordres ou des plans basilicaux, la synagogue Altneuschul de Prague est un monument gothique à deux piliers centraux comme les salles capitulaires de l'époque, la synagogue de Kaïfeng, encore attestée au XVIII ${ }^{\mathrm{e}}$ siècle, avait l'apparence d'une pagode, les synagogues de Djerba offrent des dispositions communes dans l'architecture arabe, comme des séries d'arcades ou des patios... Aussi d'une certaine manière, l'option architecturale est assez peu significative de la conception que les Juifs se faisaient eux-mêmes de la synagogue, construite d'ailleurs le plus souvent par des architectes non-juifs.

Néanmoins, dans l'histoire de l'architecture des synagogues occidentales ou construites par des communautés issues d'Europe, il convient de discerner trois phases. La première correspond au processus d'intégration décrit qui s'est poursuivi dans une grande partie du monde non-occidental jusqu'à nos jours. On pourrait aussi rattacher à cette phase les périodes où la synagogue devait obligatoirement être invisible dans l'espace urbain. La seconde voit, sous l'effet de l'émancipation, s'ouvrir la possibilité de recourir librement à un style, qui pourrait être significatif d'une conscience de soi, mais le poids du désir d'intégration nationale et sociale demeurant prépondérant, l'option la plus fréquente se trouve être les modes vernaculaires, puis, quand règne l'historicisme, le modèle des églises. L'absence de modèles de synagogues, qui du jour au lendemain pouvaient avoir pignon sur rue, a naturellement poussé les architectes démunis à s'inspirer des édifices de cultes qui leur étaient les plus familiers, les églises. La troisième phase se caractérise par une mutation générale de l'architecture qui se libère du langage des styles historiques, au profit d'une modernité plus ou moins affirmée, et par un mouvement de retour aux sources amorcé par les milieux orthodoxes, bientôt suivis par une majorité de communautés.

Si l'émancipation introduit une certaine liberté dans les choix architecturaux, l'intégration passe toujours par le recours au style architectural dominant ; ainsi les synagogues d'Amsterdam, élevées au XVII ${ }^{\mathrm{e}}$ siècle dans un contexte très libéral, n'en sont pas moins des bâtisses typiquement hollandaises. La nationalisation des Juifs, au cours du XIX ${ }^{\mathrm{e}}$ siècle, se traduit par le recours aux styles historiques 
adoptés par la majorité, comme un symbole d'allégeance aux valeurs esthétiques et identitaires qui fondent la nationalité. Quand l'identité américaine se construit en usant du néoclassicisme comme style fédéral, nombre de synagogues sont édifiées selon cette esthétique : ce qui importe alors aux Juifs américains, c'est d'assurer la promotion de la synagogue en tant que monument visible dans la ville et leur propre américanisation. Le processus est strictement concomitant aux tendances réformistes et s'observe également en Europe. La synagogue devenue un bâtiment public, au financement duquel participent de plus en plus les instances administratives de la ville ou de l'état, reçoit une forme qui repose sur des facteurs esthétiques, identitaires et sociaux, et non pas religieux. Cependant, lorsque les styles historiques tendent à se spécialiser en correspondance avec des fonctions, certains paraissent plus adéquats à la justice, à l'autorité municipale ou à l'architecture religieuse ; c'est ainsi que des synagogues néo-gothiques ou romano-byzantines voient le jour : le modèle de l'église est alors envahissant et contribue à cette sacralisation décrite précédemment.

Dans le choix des formes historiques dont la synagogue est habillée, l'imagination a plus à voir que les références juives qui serviraient à dégager sa spécificité. Les synagogues orientalistes doivent plus à une vision sublimée de l' «âge d'or » andalou ${ }^{22}$ et à l'Alhambra de Grenade qu'aux synagogues de Tolède. Et il a fallu l'engouement de quelques architectes juifs, frappés par la découverte des anciennes synagogues de Galilée, à la fin du XIX siècle, pour tenter d'y puiser des motifs juifs. On en reste de toute façon à une dimension décorative, perceptible par exemple dans la façade colossale et l'arche sainte de la synagogue Shearith Israel (1897), à New York, où Arnold Brunner emploie des couronnes empruntées aux ruines de Kfar Baram. Ces démarches traduisent une volonté de donner à la synagogue des formes perçues comme juives, mais demeurent fidèles à la logique de valorisation de la synagogue en lieu de culte, voire de sacralisation. Seuls les Juifs orthodoxes

22. D. Jarrassé, «Sefarad imaginaire : le style hispano-mauresque dans les synagogues du XIX ${ }^{\mathrm{e}}$ siècle », Mémoires juives d'Espagne et du Portugal, colloque Paris IV-Stanford, Paris, Publisud, 1996, p. 261-269. 
prirent conscience que le recours à ces types architecturaux, même inspirés de synagogues anciennes, restait en contradiction avec les fondements de la simplicité synagogale. Aussi les voit-on utiliser une architecture domestique. La célèbre synagogue de la rue Pavée, construite par Hector Guimard en 1914, en est un exemple majeur : la salle de culte, rejetée à l'arrière, ne détermine plus la façade, qui reflète en partie les étages des salles communautaires; quant à l'option «modern-style », elle est en adéquation avec l'aspiration du groupement d'associations orthodoxes, Agoudath Hakehilos, qui a commandité Guimard, puisqu'elle permet d'échapper aux références historiques, tout particulièrement à celles des styles empruntés aux églises. Cette réaction anticipe la tendance à réinscrire l'espace de culte dans un ensemble communautaire, selon la tradition qui avait perduré dans les milieux orthodoxes d'Europe de l'Est. L'ancêtre du centre communautaire, le premier Jewish Center du rabbin Mordechaï Kaplan, date de 1919; il était installé dans un building sans caractère de la $86^{\mathrm{e}}$ rue à New York et regroupait des activités religieuses, sociales, culturelles et sportives.

Une rupture importante se fait jour donc autour de 1914, mais durant toutes les années de l'entre-deux-guerres, la concurrence demeura entre des synagogues inspirées de modèles historiques et des synagogues résolument modernes, voire des centres polyvalents. Parfois deux bâtiments sont encore associés, une synagogue toujours magnifiée par une architecture prestigieuse et un « Social Center» plus modeste d'apparence le plus souvent.

Cependant la modernité est aussi une forme de normalité, une modalité d'intégration. Type non fixé par la Loi, contrairement à l'église orthodoxe par exemple, la synagogue continue de suivre les évolutions architecturales et, la fonction religieuse appelant des extrapolations symboliques audacieuses, elle a pu être l'occasion de projets extraordinaires, comme Beth Sholom édifié en 1956 par Frank Lloyd Wright à Elkins Park, près de Philadelphie ${ }^{23}$, ou Cymbalista Synagogue, due à Mario Botta et inaugurée sur le campus de l'université de Tel-Aviv en 1998. Ces édifices montrent l'ampleur

23. Il est significatif tout de même que cette synagogue très originale rappelle un projet élaboré en 1926 pour une cathédrale prévue à New York. 
de la liberté de conception laissée aux architectes : Wright a rêvé d'un mont Sinaï translucide et l'a réalisé, selon une interprétation très personnelle. Quant à la nouvelle synagogue de Tel-Aviv, elle offre une étonnante synthèse des composantes religieuse et laïque de la société israélienne, en associant dans un bâtiment bicéphale centre culturel et synagogue : deux volumes cylindriques surmontent, à l'ouest, l'auditorium du Jewish Heritage Center, et, à l'est (vers Jérusalem), une synagogue, signifiant par leur parallélisme l'égalité des aspirations séculière et religieuse des Israéliens. Le projet concilie aussi points de vue orthodoxe et réformé, car si la synagogue suit le plan traditionnel avec bima centrale, l'auditorium, polyvalent, peut aussi servir de synagogue réformée. Le choix de Mario Botta par le mécène suisse Norbert Cymbalista n'est pas indifférent, car cet architecte, dont on a montré la « vocation pour le sacré et le monumental ${ }^{24}$, a traité plusieurs programmes chrétiens, comme la cathédrale d'Evry (1995), et son langage tend évidemment à assimiler la synagogue aux autres édifices de culte, voire aux musées, cet autre lieu consacré de la culture contemporaine. Il en va de même avec Chagall, dont la capacité à passer de la cathédrale à la synagogue, semble attester l'existence d'un « Art Sacré » ${ }^{25}$, au-delà des différentes confessions : est-ce sa fréquentation du milieu animé par le Père Marie-Alain Couturier, le rénovateur de l'art chrétien, qui lui a inspiré l'usage de cette expression lors de l'inauguration des vitraux de l'hôpital Hadassah de Jérusalem (1962) ? Dès qu'une œuvre au service de la spiritualité atteint un haut degré de qualité esthétique, et ici Chagall réalise un des chefs-d'œuvre de l'art religieux du $\mathrm{XX}^{\mathrm{e}}$ siècle, elle semble chargée de « sacré ». La synagogue n'échappe pas à cette tentation; certes, elle ne singe plus pour cela la cathédrale, mais en sollicitant les artistes, elle ne peut empêcher que cela soit perçu comme une forme de sacralité.

24. Werner Oechslin, « Mario Botta - Une Vocation pour le sacré et le monumental », dans Mario Botta. Bâtiments publics 1990-1998, Milan, Skira/ Le Seuil, 1998, p. 26-30.

25. Allocution citée dans Sylvie Forestier, Marc Chagall. L'œuvre monumental. Les Vitraux, Milan, Jaca Book, 1987, p. 185. 
Cependant un fait atteste clairement le rejet de la sacralisation de la synagogue, parallèlement d'ailleurs à l'abandon de l'israélitisme et de son expression majeure qu'est le «temple », la prédominance dans le judaïsme contemporain du centre communautaire. À côté de grandes synagogues, à valeur essentiellement symbolique, édifiées pour marquer la renaissance du judaïsme après la Choa (Strasbourg, Livourne, Allemagne) ou son épanouissement sur le sol américain, ce sont les centres communautaires, où sont associées des fonctions sociales, culturelles et ludiques, qui ont la priorité ; néanmoins, cela n'empêche pas les architectes de distinguer la partie cultuelle par des formes ou des motifs symboliques. Ainsi Erich Mendelsohn qui a donné une impulsion essentielle à ce type d'édifice dans les années 1950 aux États-Unis, a continué à réserver à l'espace synagogal un traitement particulier, jouant souvent sur des volumes différenciés. Le refus de la sacralisation ne saurait, pour d'évidentes raisons de respect, réduire la salle de culte au rang d'un gymnase. Le judaïsme ne peut donc totalement renier cette hiérarchisation et cet héritage de l'âge des émancipations qui vit la promotion de la synagogue, «sacralisée » non pas dans sa nature même, mais dans ses formes. Les historiens de l'art seraient presque excusables de vouloir y déceler un «art sacré »... Néanmoins, parallèlement à ce maintien des lieux de cultes tentés par les signes extérieurs d'une représentativité, la prolifération des oratoires, discrets ou cachés, montre, dans le judaïsme contemporain, le retour en force du modèle du bet hamidrach et la prédominance de l'étude sur le culte chargé de décorum.

d.jarrasse@free.fr 


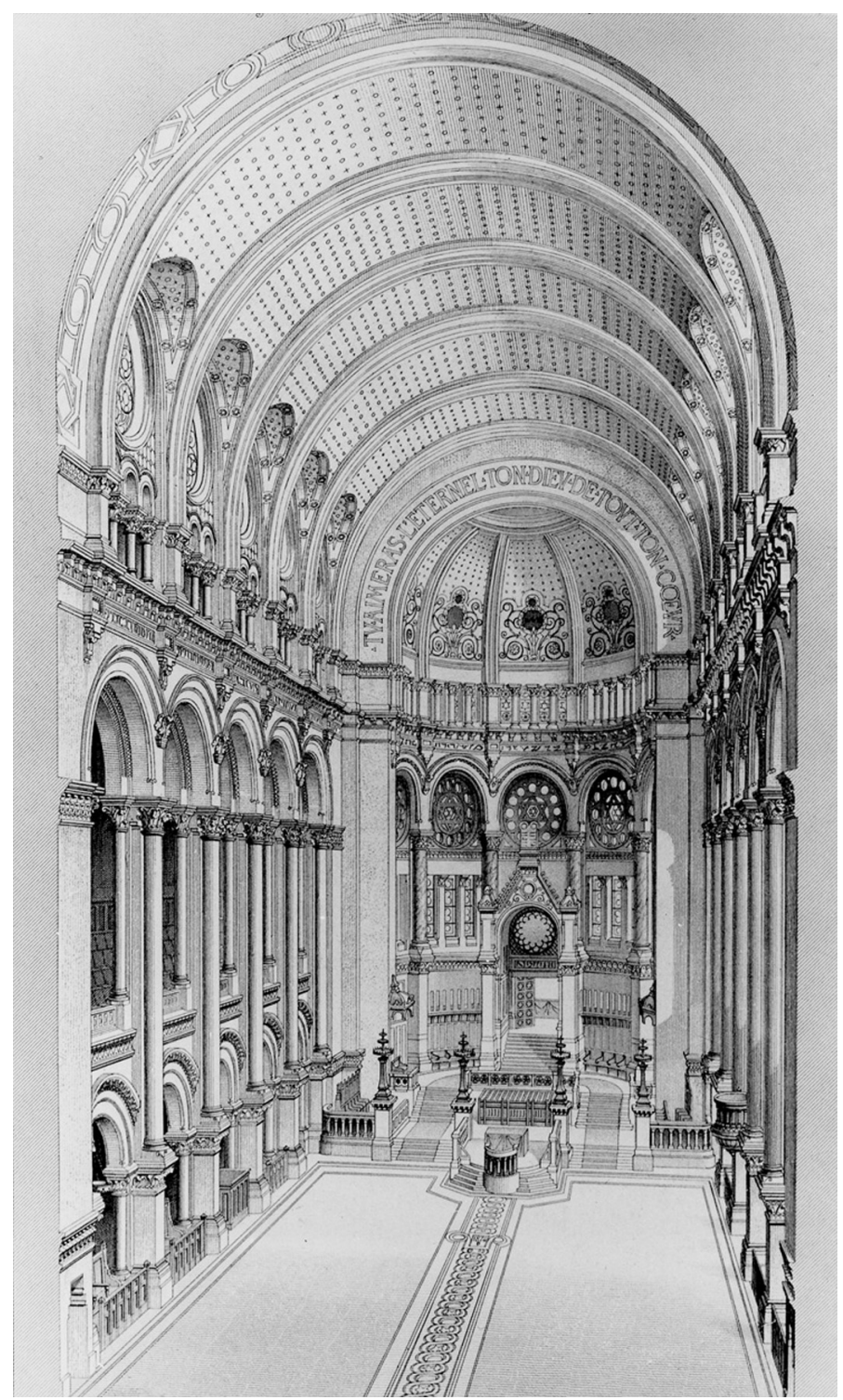

Alfred AldRophe, architecte de la ville de Paris et du consistoire israélite (1854-1895). Synagogue de la rue de la Victoire à Paris. Vue intérieure. Gravé dans F. Narjoux, Monuments élevés par la ville, 1850-1880. D. R. 
